\title{
Performance Analysis and control strategies of Cascaded Multilevel Converters
}

\author{
Polu Veera Pratap ${ }^{1}$, S. Sridhar ${ }^{2}$ \\ ${ }^{1}$ Department of Electrical and Electronics Engineering, Jntua College of Engineering, Anantapur, Andhra \\ Pradesh, India \\ ${ }^{2}$ Assistant Professor, Department of Electrical and Electronics Engineering, Jntua college of Engineering, \\ Andhra Pradesh, India
}

\begin{abstract}
Multilevel inverters have been widely used for high-voltage and high-power applications. Their perf0rmance is greatly superi0r t0 that of c0nventi0nal tw0-level inverters due t0 their reduced t0tal harm0nic dist0rti0n (THD),. This t0p0l0gy requires fewer c0mp0nents when c0mpared t0 di0de clamped, flying capacit0r and Bridgeless cascaded inverters and it requires fewer carrier signals and gate drives. Theref0re, the 0verall c0st and circuit c0mplexity are greatly reduced. This paper presents a n0vel reference and multicarrier based PWM scheme It als0 c0mpares the perf0rmance of the pr0p0sed scheme with that Of c0nventi0nal cascaded bridge less rectifier (CBR) multilevel inverters. finally Simulati0n results fr0m MATLAB/SIMULINK are presented t0 verify the perf0rmance of the Five-level Multilevel Inverter
\end{abstract}

Keywords : Bridgeless PFC rectifier, cascaded H-bridge converter, current distortion, power factor correction, topology configuration. Modulation Index (MI).

\section{INTRODUCTION}

Multilevel p0wer c0nversi0n was first intr0duced m0re than tw0 decades ag0. The general c0ncept inv0lves utilizing a higher number of active semic0nduct0r switches t0 perf0rm p0wer c0nversi0n in small v0ltage steps. There are several advantages t0 this appr0ach when c0mpared with the c0nventi0nal p0wer c0nversi0n appr0ach. The smaller v0ltage steps lead t0 the production of higher p0wer quality wavef0rms, and they reduce b0th the v0ltage $(d v / d t)$ stress $0 \mathrm{n}$ the 10ad and the electr0magnetic c0mpatibility c0ncerns [1]. An0ther imp0rtant feature of multilevel inverters is that their semic0nduct0rs are wired in a series-type c0nnecti0n, which all0ws 0perati0n at higher v0ltages. H0wever, this series eliminates 0verv0ltage c0ncerns.
Furtherm0re, since the switches are n0t truly series c0nnected, their switching can be staggered.

This reduces the switching frequency which reduces the switching 10sses. With the advancement of p0wer semic0nduct0r devices and 0ther p0wer electr0nicsrelated techn0l0gies, the emerging c0ncept of the transf0rmer less cascaded multilevel c0nverter (TCMC) has rapidly devel0ped and caught increasing attenti0n fr0m b0th the academia and industry in the past decades [1]-[9]. It is able realize direct c0nnecti0n t0 the high v0ltage with 0ut inv0lving a bulky and line frequency transf0rmer.

This eventually reduces the system reliability and increases the implementati0n c0sts [12]-[14]. H0wever, in nearly $70 \%$ of practical applicati0ns, including speed regulation f0r pumps, wind p0wer 
integrati0n, and plug-in electrical vehicle applicati0ns, 0nly a unidirecti0nal p0wer flow is required [15]-[17]. F0r such practices, s0me fully c0ntrolled switches can be eliminated $0 \mathrm{r}$ replaced t0 simplify the system [18]. R0nan et al. [19], [20] pr0p0sed a cascaded b00st PFC rectifier. As can be seen fr0m Fig. 2, several p0wer m0dules are cascaded for direct c0nnecti0n t0 the medium v0ltage grid.

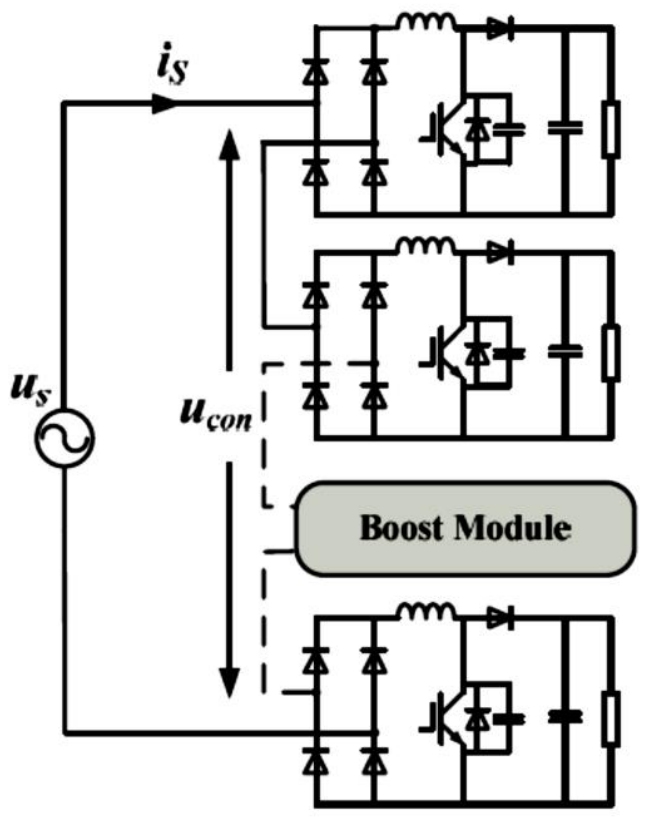

Fig. 1. T0p0logy of single-phase cascaded diode Hbridge rectifier.

Each of these power modules is composed of a traditi0nal b00st PFC circuit. In this way, the number of fully c0ntrolled switches is greatly reduced. H0wever, the b00st induct0r Of each p0wer m0dule is 10cated at the dc side. Under certain c0nditions, the energy st0red in the b00st induct0r can generate a circulating current that circulates through the fully c0ntrolled switch and the diOdes. COnsequently, the large circulating current may damage the devices $0 \mathrm{r}$ even break d0wn the wh0le system. Additi0nally, the p0wer m0dule sh0rted by the circulating current is actually bypassed from the p0wer grid, thus the grid v0ltage has t0 be shared am0ng the 0ther cascaded m0dules. As a result, the v0ltage stresses of the switches in th0se m0dules increase greatly [21]. An0ther problem is that the current is always carried thr0ugh three semic0nduct0r devices within each m0dule, causing relatively high c0nducti0n 10sses [22]. Reference [23] presented It is w0rth p0inting Out that a three-phase multilevel rectifier uses three times as many cascaded m0dules as a same rated single-phase rectifier d0es. Theref0re, devel0ping a new t0p010gy f0r the three-phase cascaded multilevel rectifier brings even m0re attractive benefits. H0wever, n0 related research has been rep0rted yet. This paper presents a cascaded bridgeless multilevel rectifier (CBR) aiming at using fewer fully c0ntrolled switches t0 reduce hardware c0mplexity, increase system reliability, and cut d0wn the implementation expenses. Based 0n analyzing the physical cause of the input current zer0-cr0ssing dist0rti0n when the single-phase CBR is 0perating under a unity p0wer fact0r, an impr0ved c0ntr0l strategy is pr0p0sed t0 achieve a satisfact0ry p0wer fact0r and eliminate the input current zer0-cr0ssing dist0rti0n. Besides, a revised t0p010gy of the single-phase CBR is presented as an0ther s0luti0n f0r av0iding the input current dist0rti0n under the unity p0wer fact0r c0nditi0n. In additi0n, different fr0m the single-phase case, the three-phase CBR can achieve a unity p0wer fact0r with greatly attenuated input current zer0-cr0ssing dist0rti0n by empl0ying the traditi0nal c0ntr0l meth0d.

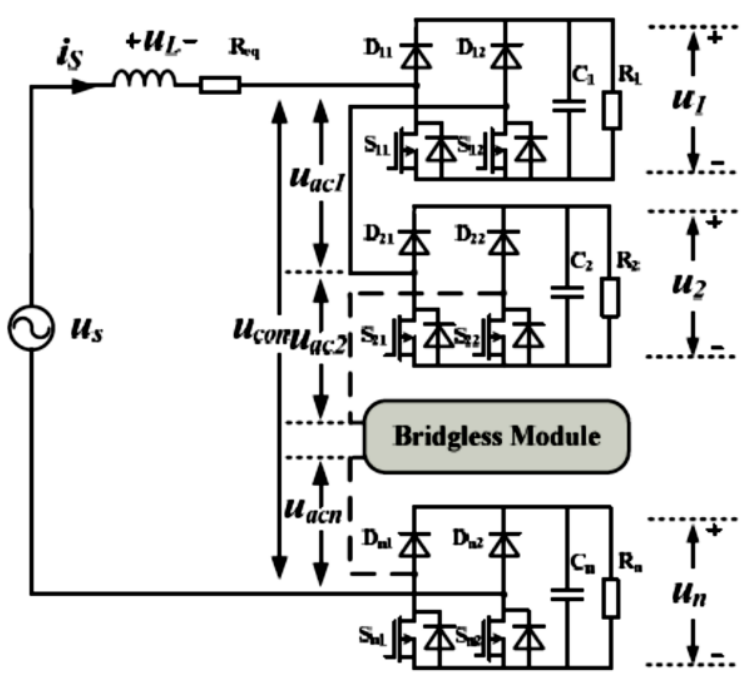

Fig 2. Topology of single-phase CBR. 
The rest Of this paper is Organized as f0ll0ws. Secti0n II presents the COnventi0nal On this basis, tw0 s0luti0ns f0r achieving a satisfact0ry p0wer fact0r and eliminating the input current dist0rti0n are pr0p0sed In this paper mainly p0wer fact0r by limiting the maximum value of the b00st inductance. As stated ab0ve, the impr0ved c0ntr0l strategy leads t0 a lagging p0wer fact0r that is cl0sely related to the b00st inductance. Theref0re, by pr0perly limiting the maximum b00st inductance, the p0wer fact0r can be c0ntr0lled ab0ve the minimum acceptable value. Define $\mathrm{k}$ as the minimum p0wer fact0r all0wed. Substituting c0s $\phi=\mathrm{k}$ int0 (8) yields the limitation of the maximum b00st inductance.

$$
L_{\max }=\frac{U_{S}^{2} \sin \left(2 \cos ^{-1} k\right)}{2 \omega U_{d}^{2} \sum_{i=1}^{n} \frac{1}{R_{i}}} .
$$

\section{PROPOSED CASCADED MULTI LEVEL INVERTER}

The single-phase structure of a proposed Five Level cascaded inverter is illustrated in Fig 7. Each separate $\mathrm{dc}$ s0urce is c0nnected t 0 a single-phase full-bridge $0 \mathrm{r}$ $\mathrm{H}$-bridge inverter. Each inverter level can generate three different v0ltage Outputs, $+\mathrm{Vdc}, 0$ and $-\mathrm{Vdc}$, by c0nnecting the dc s0urce t0 the ac Output with different switching c0mbinations of the four semic0nduct0r switches $\mathrm{T} 1, \mathrm{~T} 2, \mathrm{~T} 3$ and $\mathrm{T} 4$. T0 0btain +Vdc, switches T1 and T2 are tuned 0n, while -Vdc can be Obtained by tuning 0n switches T3 and T4. By turning 0n T1 and T3 0r T2 and T4, the Output v0ltage is 0 . The ac 0utputs of each of the full-bridge inverter levels are c0nnected in series such that the synthesized v0ltage wavef0rm is the sum of the inverter Outputs [20], [21].

$$
\begin{aligned}
& \mathrm{m}=2 \mathrm{n}+\mathbf{1} \\
& \mathrm{N}=2(\mathrm{~m}-1)
\end{aligned}
$$

Where $\mathrm{m}$ is the number of levels, $\mathrm{n}$ is the number of DC s0urces, and $\mathrm{N}$ is the number Of switching devices in each phase. The m0st well-kn0wn SPWM which can be applied t0 a pr0p0sed cascaded multilevel inverter (CCMLI) is the Phase-Shifted SPWM. This m0dulati0n technique is alm0st the same as the c0nventi0nal SPWM technique which is applied t0 a c0nventi0nal single phase bridgeless inverter. The Only difference between them is that the PhaseShifted SPWM utilizes m0re than 0ne carrier. The number of carriers used per phase is equal t0 twice the number of dc v0ltage s0urces per phase (2n) [20].

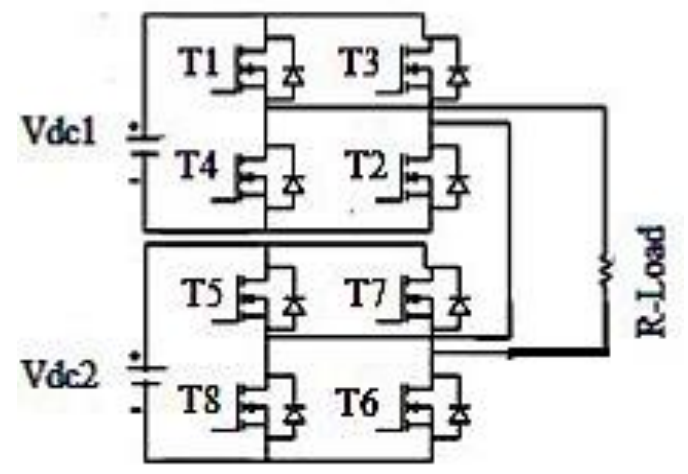

Fig 3. five level cascaded inverter Cascaded five level inverters

\section{Working Operation of Five Level Inverter:}

The working operation of cascaded $\mathrm{H}$ bridge five levels multilevel is explained below:

Mode1:-In this mode of 0perati0n single phase five level cascaded H-Bridge multilevel inverter switch1;switch3,switch5 and switch7 are turned 0n with0ut c0nnecting s0urce t0 the 10ad. The 0utput v0ltage acr0ss the 10ad 0btained is zer0.

M0de2:- In this m0de of 0perati0n single phase five level cascaded H-Bridge multilevel inverter switch1,switch3,switch5 and switch8 are turned 0n.The Output v0ltage acr0ss the 10ad Obtained is +Vdc2.

M0de3:-In this m0de of 0peration single phase five level cascaded $\mathrm{H}$-Bridge multilevel inverter

switch1,switch4, switch5 and switch8 are turned 0n.The Output v0ltage acr0ss the 10ad Obtained is $V d c 1+V d c 2$.

M0de4:-In this m0de of 0peration single phase five level H-Bridge cascaded multilevel inverter 
switch2,switch4,switch6 and switch7 are turned 0n.The Output v0ltage acr0ss the 10ad Obtained is Vdc2.

M0de5:-In this m0de of 0perati0n single phase five level $\mathrm{H}$-Bridge cascaded multilevel inverter switch2, switch4,switch6 and switch8 are turned 0n. The Output v0ltage acr0ss the 10ad Obtained is zer0.

M0de6:- In this m0de of 0perati0n single phase five level $\mathrm{H}$-Bridge cascaded multilevel inverter switch3,switch2,switch7 and switch6 are turned 0n.The Output v0ltage acr0ss the 10ad 0btained is Vdc1-Vdc2

\begin{tabular}{|l|l|l|l|l|l|l|l|l|}
\hline M0de & S1 & S2 & S3 & S4 & S5 & S6 & S7 & S8 \\
\hline 1 & 1 & 0 & 1 & 0 & 1 & 0 & 1 & 0 \\
\hline 2 & 1 & 0 & 1 & 0 & 1 & 0 & 0 & 1 \\
\hline 3 & 1 & 0 & 0 & 1 & 1 & 0 & 0 & 1 \\
\hline 4 & 0 & 1 & 0 & 1 & 0 & 1 & 1 & 0 \\
\hline 5 & 0 & 1 & 0 & 1 & 0 & 1 & 0 & 1 \\
\hline 6 & 0 & 1 & 1 & 0 & 0 & 1 & 1 & 0 \\
\hline
\end{tabular}

Fig. 4. Single phase structure of the Pr0posed cascaded multilevel inverter.

\section{MODULATION TECHNIQUE}

Pulse Width Modulation (PWM) control strategydevelopment tries to reduce the total harmonic dist0rti0n (THD) of the Output v0ltage. Increasing the switching frequency of the PWM pattern reduces the 10wer frequency harm0nics by m0ving the switching frequency carrier harm0nic and the ass0ciated sideband harm0nics away from the fundamental frequency c0mp0nent [21]. This increased switching frequency reduces harm0nics. This results in a 10wer THD with high quality 0utput v0ltage wavef0rms of the desired fundamental RMS value and frequency, which are as cl0se as p0ssible t0 the sinus0idal wave shape. Any deviation fr0m the sinus0idal wave shape will result in harm0nic currents in the 10ad and this harm0nic current pr0duces electr0magnetic interference (EMI), harm0nic 10sses and t0rque pulsation in the case of m0t0r drives. A higher switching frequency can be empl0yed f0r 10w and medium p0wer inverters. Meanwhile, f0r high p0wer and medium v0ltage applications the switching frequency sh0uld be $10 \mathrm{w}$. Harm0nic reducti0n can then be strictly related t0 the perf0rmance of an inverter with any switching strategy. Three phase multilevel inverters require three m0dulating signals $0 \mathrm{r}$ reference signals which are three-unip0lar sine waves with a 120 degree phase shift. In this paper, three new carrier based PWM techniques are devel0ped as follows:

\section{Triangular Multicarrier Unip0lar Sine PWM (TMC USPWM)}

2. Saw T00th Multicarrier Unip0lar Sine PWM (STMC USPWM)

3. Unip0lar Sine Multicarrier Unip0lar Sine PWM (USMC USPWM)

Each carrier is c0mpared with a corresponding modulating uni p0lar sine wave. The reference $0 \mathrm{r}$ m0dulati0n wavef0rm has peak amplitude Am and a frequency $\mathrm{fm}$, and it is centered in the middle of the carrier set. The general principle of the carrier based PWM technique is a c0mparis0n of a reference wavef0rm with a carrier wavef0rm, this typically being a triangular carrier wavef0rm. The reference is c0ntinu0usly c0mpared with the carrier signal. If the reference is greater than the carrier signal, then the active device c0rresp0nding t0 that carrier is switched $0 \mathrm{n}$, and if the reference is less than the carrier signal, then the active device c0rresp0nding t0 that carrier is switched 0ff. The carrier frequency defines the switching frequency of the c0nverter and the high Order harm0nic c0mp0nents of the Output v0ltage spectrum. and t-he sidebands 0ccur ar0und the carrier frequency and its multiples. In multilevel inverters, the amplitude m0dulation index, $\mathrm{Ma}$, and the frequency rati0, Mf, are defined as: 


$$
\begin{gathered}
M_{a}=\frac{A_{r}}{((m-1) / 2) A_{c}} \\
M_{f}=\frac{f_{c}}{f_{r}}
\end{gathered}
$$

Where Ar and Ac are the amplitude of the reference and the carrier signal, respectively.fr and fc are the frequency of the reference and the carrier signal respectively[22]. In this paper, the m0dulati0n indexes used are 0.8, 0.9 and A vari0us pulse width 10sses and THD, pulse width m0dulati0n(PWM) techniques have been discussed t0 c0ntr0l the inverter[3]. due t0 their unique characteristics such as directly using the c0ntr0l variable, impr0ving DC link v0ltage utilizati0n, reducing c0mmutati0n.

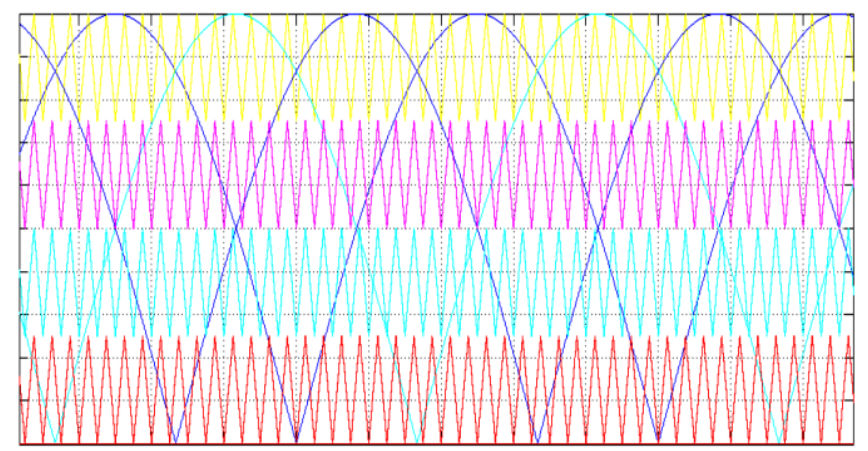

(a)

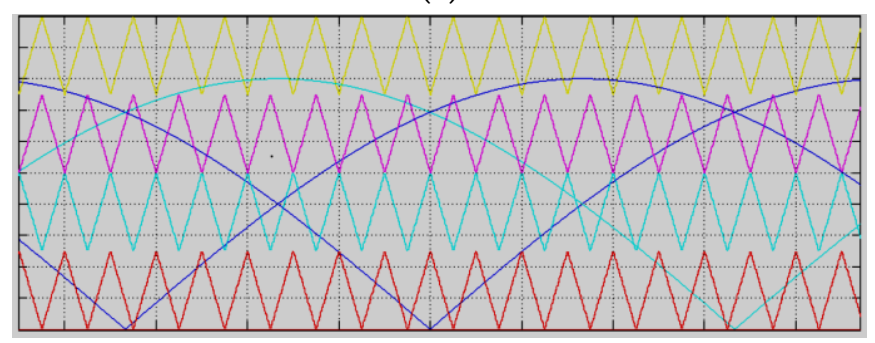

(b)

Fig 5. PWM for five level inverter with modulation index $=0.8$

\section{ANALYSIS OF SYSTEM}

For the pr0p0sed current c0ntr0lled inverter or rectifier, the switching requirement can be stated as f0llows. Given a desired set of three phase v0ltages and a set of three phase Currents f0r the Output inverter

$$
\begin{aligned}
& \mathrm{V}_{\mathrm{a}}=\mathrm{V} \sin w t \\
& \mathrm{~V}_{\mathrm{b}}=\mathrm{V} \sin \left(w t-\frac{2 \pi}{3}\right) \\
& \mathrm{V}_{\mathrm{c}}=\mathrm{V} \sin \left(w t+\frac{2 \pi}{3}\right) \\
& \mathrm{I}_{\mathrm{a}}=\mathrm{I} \sin (w t-\alpha) \\
& \mathrm{I}_{\mathrm{b}}=\mathrm{I} \sin \left(w t-\alpha-\frac{2 \pi}{3}\right) \\
& \mathrm{I}_{\mathrm{c}}=\mathrm{I} \sin \left(w t-\alpha+\frac{2 \pi}{3}\right)
\end{aligned}
$$

Where $\mathrm{V}$ and I are v0ltage and currents, respectively. Determine the switching functi0n [S] that will produce a desired set of line -gr0und v0ltages

$$
\left[\begin{array}{l}
V_{1 n} \\
V_{2 n}
\end{array}\right]=\left[\begin{array}{ll}
S_{1} & S_{2} \\
S_{3} & S_{4}
\end{array}\right]\left[\begin{array}{c}
V_{d c} \\
-V_{d c}
\end{array}\right]
$$

$\mathrm{S} 1+\mathrm{S} 2=1$

$\mathrm{S}_{3}+\mathrm{S}_{4}=1$

$0 \leq \mathrm{S}_{\mathrm{n}} \leq 1$

Where $\mathrm{n}=1 \ldots . .4$

$\mathrm{V}_{1 \mathrm{n}}=\mathrm{V}_{1}-\mathrm{V}_{3}=\sqrt{3} \mathrm{~V}_{\mathrm{dc}} \sin \left(w t-\frac{\pi}{6}\right)$

$\mathrm{V}_{2 \mathrm{n}}=\mathrm{V}_{2 \mathrm{n}}=\sqrt{3} \mathrm{~V}_{\mathrm{dc}} \sin \left(w t-\frac{\mathrm{m}}{2}\right)$

where, $\boldsymbol{n}$ is the dc bus centre p0int assumed t0 be gr0und .Here, the dc capacit0r v0ltages vcl and vc2 are assumed to be $V_{\text {dc. }}$. One can c0nfirm that the phase difference is $60 "$.The ab0ve equati0n can be s0lved as follows

$$
\begin{aligned}
& \mathrm{s}_{1}=0.5\left[1+\mathrm{a} 0 \sin \left(w t-\frac{\pi}{6}\right)\right] \\
& \mathrm{s}_{2}=0.5\left[1-\mathrm{a} 0 \sin \left(w t-\frac{\pi}{6}\right)\right] \\
& \mathrm{s}_{3}=0.5\left[1+\mathrm{a} 0 \sin \left(w t+\frac{\pi}{2}\right)\right] \\
& \mathrm{s}_{4}=0.5\left[1-\operatorname{aos} \sin \left(w t-\frac{\pi}{2}\right)\right]
\end{aligned}
$$

where $\mathrm{a} 0=\sqrt{3} \frac{V}{V_{d c}}, \quad\left|a_{0}\right| \leq 1$

The 0verall dc link v0ltage can be maintained c0nstant by the dc link v0ltage c0ntr0ller which makes the sum of charging currents zer0 by c0ntr0lling the magnitude of individual capacit0r v0ltages fluctuate and depend $0 n$ the Operating frequency, capacitance ,and the magnitude of current. 


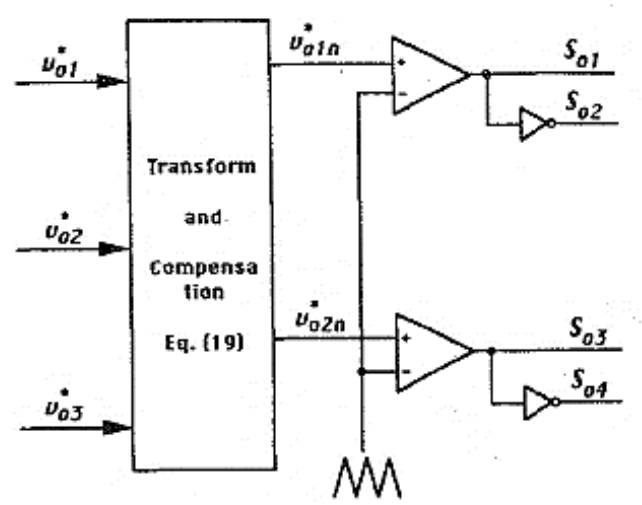

Fig 6. Sine triangular pulse width modulation

These voltage references are compared by triangular modulation signal to generate the gating signals for two legs of Inverters Because the input references are in phase with the input phase voltages, the PI controller output is, in effect, the magnitude of power component of current the required power by the capacit0rs t0 maintain the dc link v0ltage c0nstant. Reactive current c0mp0nents can be added to the current references t0 c0ntr0l the input p0wer fact0r.

VariOus current c0ntrOllers can be applied t0 the c0ntr01 of this system. F0r the ramp c0mparis0n meth0d t0 c0ntr0l the switching frequency, the current reference is c0mpared with the actual measured current t0 calculate the current err0r, which are passed thrOugh the current c0ntr0ller, usually PI c0ntr0ller, to generate the required PWM v0ltages at the rectifier Output

\section{CONTROL STRATEGY OF THE MULTILEVEL RECTIFIERS}

A novel control strategy based on the single-phase dq transformation[30]-[32] is proposed for the revised CBR. the control block diagram. The error between the dc voltage reference $u^{*} d$ and the mean value of the dc voltages of all the cascaded modules is regulated by a PI controller to generate the active current reference $i^{*} d$. Meanwhile, the reactive current reference $i^{*} q$ is set to be zero in order to achieve the unity power factor. Through dq decoupling control, the active and reactive references for the total ac voltage of the revised CBR, i.e., $u^{*}$ cond and $u^{*}$ conq, are obtained. According to (10), the ac voltage references $u^{*} \mathrm{AB}$ and $u^{*} \mathrm{BC}$ should meet

$$
\begin{aligned}
& \mathrm{u}^{*}{ }_{\text {cond }}=\mathrm{u}^{*}{ }_{\mathrm{ABd}}+\mathrm{u}^{*}{ }_{\mathrm{BCd}} \\
& \mathrm{u}^{*}{ }_{\text {conq }}=\mathrm{u}^{*}{ }_{\mathrm{ABq}}+\mathrm{u}^{*}{ }_{\mathrm{BCq}}
\end{aligned}
$$

In order to avoid input current zero-crossing distortion, $u \mathrm{AB}$ needs to be always in phase with $u S$. Therefore, as shown in (28), the reactive voltage reference $u^{*} \mathrm{ABq}$ is set to be zero. As a consequence, $u^{*} \mathrm{BCq}$ has to be $u^{*}$ conq. The output dc voltages should be balanced. Otherwise, the unbalanced voltage may result in capacitor overvoltage. Hence, the active power should be equally distributed among all the cascaded modules. Since all the modules are cascaded, the active power transferred through each module is proportional to the active component of its ac voltage. Therefore, the active voltage references $u^{*} \mathrm{ABd}$ and $u^{*} \mathrm{BCd}$ should be determined as

$$
\begin{aligned}
& \mathrm{u}^{*}{ }_{\mathrm{ABq}}=\mathbf{0} \\
& \mathbf{u}^{*}{ }_{\mathrm{BC}}=\mathrm{u}^{*}{ }_{\text {conq }} \\
& \mathbf{u}^{*}{ }_{\mathrm{ABd}}=\frac{\boldsymbol{n}}{\boldsymbol{m}+\boldsymbol{n}} \mathbf{u}^{*}{ }_{\text {cond }} \\
& \mathrm{u}^{*}{ }_{\mathrm{BCd}}=\frac{\boldsymbol{m}}{\boldsymbol{n + m}} \mathrm{u}^{*} \text { cond }
\end{aligned}
$$

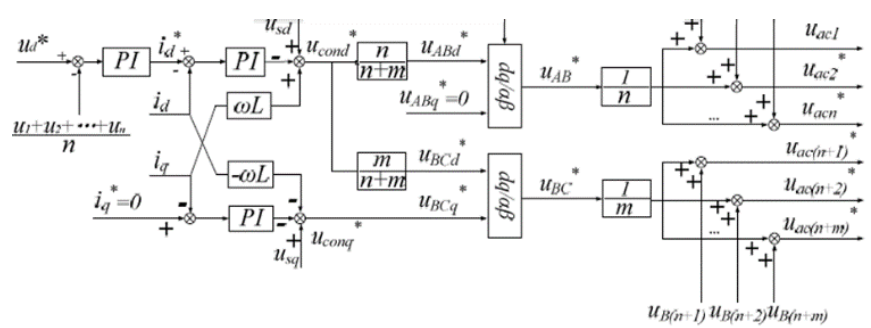

Fig 7. Control strategy based on the single-phase dq transformation 


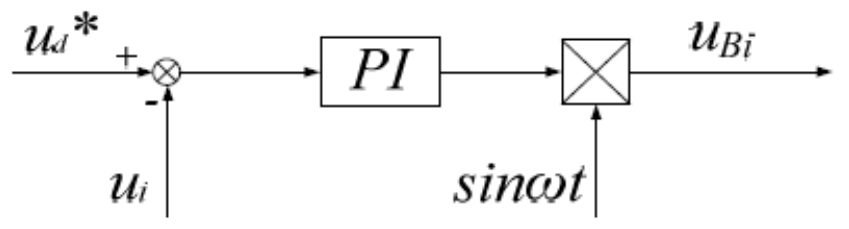

Fig 8. Diagram of output dc voltage balancing By employing two single-phase inverse $\mathrm{dq}$ transformations ,the ac voltage references, $u * \mathrm{AB}$ and $u * \mathrm{BC}$, are both obtained .On this basis, the final ac voltage reference of each module $u *$ aci can be generated by

$$
\mathrm{u}^{*} \text { aci }=\frac{u * A B d}{n}+\mathrm{uBi} \quad \mathrm{i}=1,2,
$$

the dc voltage balancing control diagram [33], [34]. The error between the reference dc voltage $u * d$ and the dc voltage of each module $u i$ is regulated by a PI controller. The output of the PI controller is then multiplied by "sin $\omega t$ " to produce the balancing signal $u \mathrm{Bi}$. As indicated in (30), the final ac voltage reference $u *$ aci is able to balance the dc voltages due to the inclusion of $u \mathrm{Bi}$.

Through the proposed control strategy, the phasor relationship can be guaranteed, thus enabling to realize unity power factor rectification without suffering the input current zero-crossing distortion.

\section{SIMULATION PARAMETERS FOR THE SINGLE-PHASE CBR AND FIVE LEVEL CHR}

\begin{tabular}{|c|c|c|}
\hline Parameter & Quantity & Values \\
\hline$u_{S}$ & input voltage & $220 \mathrm{~V}$ \\
\hline$u_{1}$ & $\begin{array}{l}\text { output DC voltage } \\
\text { of Module } 1\end{array}$ & $300 \mathrm{~V}$ \\
\hline$u_{2}$ & $\begin{array}{l}\text { output DC voltage } \\
\text { of Module } 2\end{array}$ & $300 \mathrm{~V}$ \\
\hline$f_{s}$ & $\begin{array}{l}\text { switching } \\
\text { frequency }\end{array}$ & $10 \mathrm{kHz}$ \\
\hline$L$ & boost inductance & $1.5 \mathrm{mH}$ \\
\hline$R_{l}$ & $\begin{array}{l}\text { load resistance of } \\
\text { Module } 1\end{array}$ & $20 \Omega$ \\
\hline$R_{2}$ & $\begin{array}{l}\text { load resistance of } \\
\text { Module } 2\end{array}$ & $20 \Omega$ \\
\hline$C_{l}$ & $\begin{array}{l}\text { DC capacitance of } \\
\text { Module } 1\end{array}$ & $2200 \mu \mathrm{F}$ \\
\hline$C_{2}$ & $\begin{array}{c}\text { DC capacitance of } \\
\text { Module } 2\end{array}$ & $2200 \mu \mathrm{F}$ \\
\hline
\end{tabular}

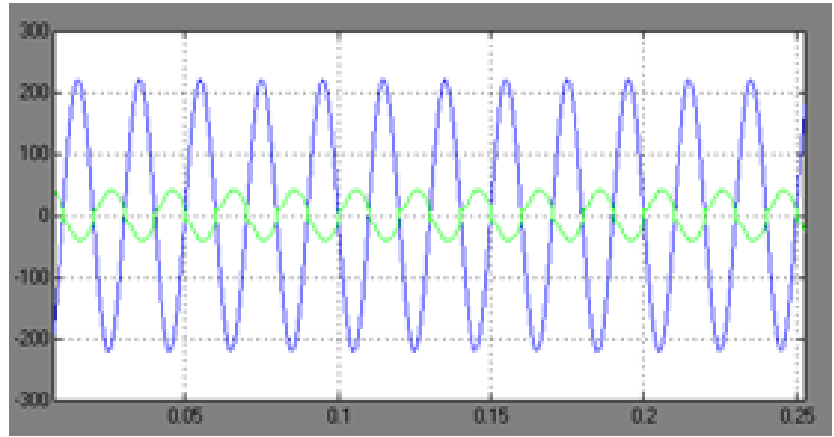

Fig 9. Input current and input voltage of the singlephase CBR under the traditional control.

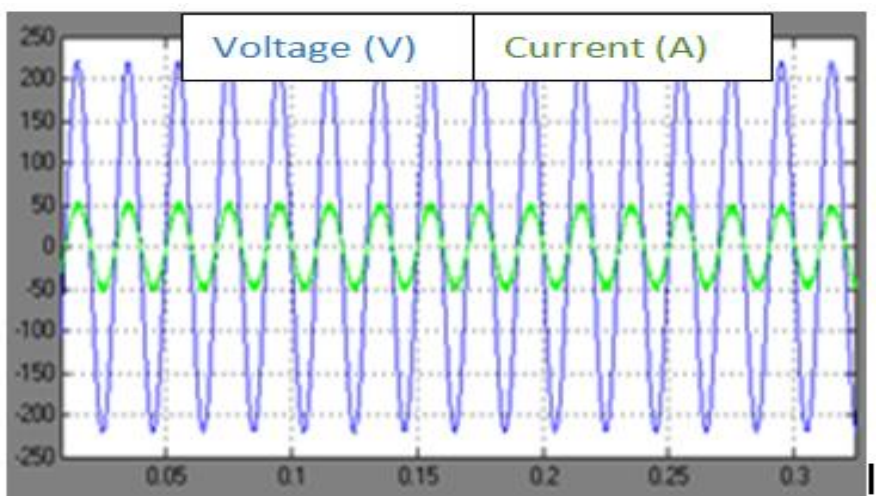

Fig 10. Input current and input voltage of the singlephase CBR under the improved control.

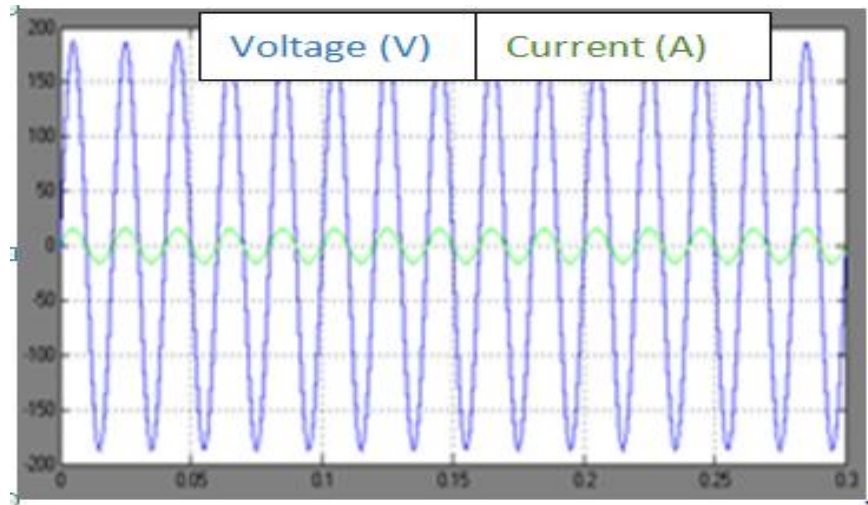

Fig 11. Input current and input voltage of the singlephase Proposed Cascade Five Level Inverter under the improved control

Fig. 9 shows the input voltage and input current of the single-phase CBR under. the traditional control, Which requires the unity p0wer fact $0 \mathrm{r}$ t 0 be achieved? The input current is in phase with the input v0ltage. H0wever, severe current dist0rti0n appears at the zer0-cr0ssings. T0 eliminate the undesirable dist0rti0n, the impr0ved c0ntr0l strategy is then empl0yed. As can be seen fr0m Fig. 10, by making 
the input current lag the input v0ltage by $\phi$, the input current dist0rti0n are av0ided. Fig. 11indicates the input current \& V0ltage of the pr0p0sed cascade five- level inverter. Fig 12 further indicates that under the impr0ved c0ntr0l, dc v0ltages of the tw0 cascaded m0dules are well balanced, which guarantees the safe and stable 0peration of the rectifier system. If a unity p0wer fact0r is strictly required, the impr0ved c0ntr0l strategy cann0t be used. Under this circumstance, the pr0p0sed cascade Five level t0p010gy is an alternative. T0 test the performance of the cascade five level, Module 1 of the Original CBR m0del is replaced by an H-bridge m0duleand the pr0p0sed c0ntr0l strategy based 0n the single-phase dq transf0rmati0n is ad0pted. That the unity p0wer fact0r is achieved with0ut causing input current dist0rti0n. Besides, Fig. 12 sh0ws that the dc v0ltages of the tw0 cascaded m0dules are regulated equal t0 each 0ther. The wavef0rms of the ac v0ltages $\mathrm{UAB}$ and $\mathrm{uBC}$ of the cascade five level are sh0wn in Fig. 12. uAB is Of a five-level f0rm due t0 the unidirecti0nal c0nduction property of the bridgeless m0dules. Since bip0lar PWM is ad0pted f0r the H-bridge m0dules, uBC has a Four-level wavef0rm.

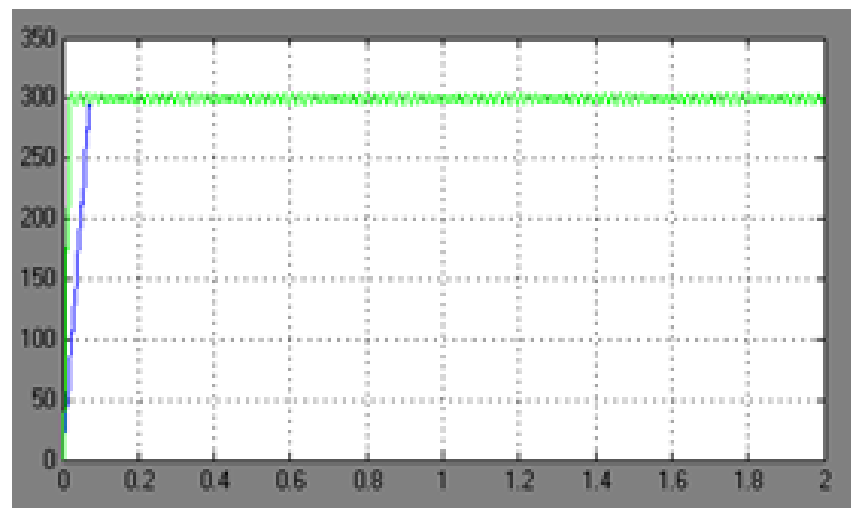

Fig 12. Output dc voltages of the single-phase CBR under the improved control.

The high-levels and low-levels voltages of b0th the ac wavef0rms are +300 +150and $-150-300 \mathrm{~V}$, respectively, dem0nstrating that the Output dc v0ltages are regulated at the reference value. T0 get an insight of the phase relati0nship, sec0nd Order $10 \mathrm{w}$-pass filters are used t0 rem0ve the high frequency harm0nics of the ac v0ltages. Fig. 14 sh0ws the three-phase currents wavef0rms. The unity p0wer fact0r is achieved. As presented in Fig. 15, a THD of $0.66 \%$ indicates an acceptable current quality shown infig16

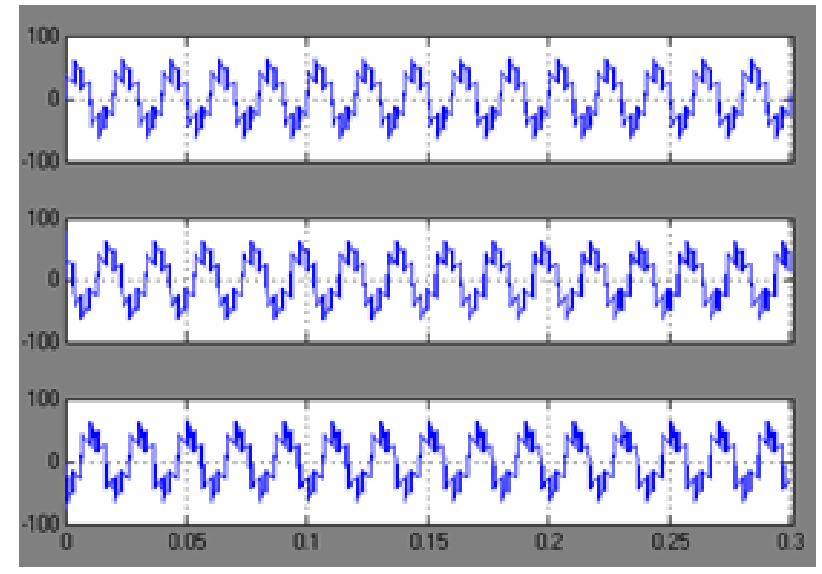

Fig 13. Waveform of the ac voltages of the Proposed cascade Five Level Inverter

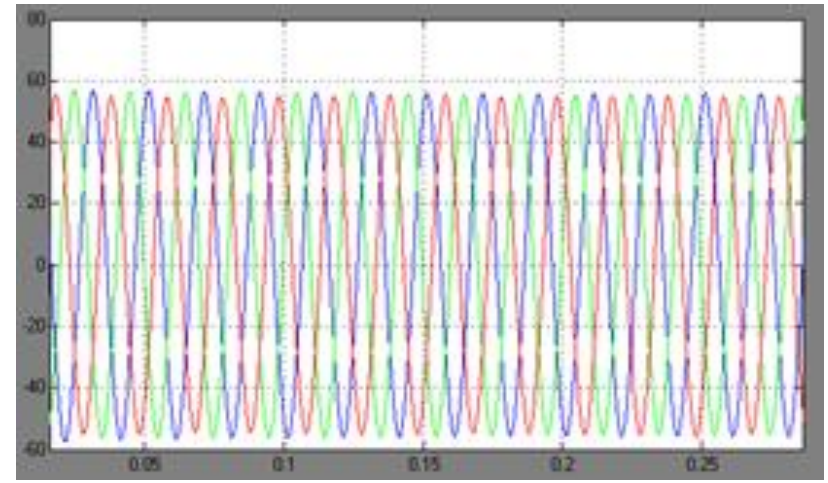

Fig 14. Waveform of the ac currents of the Proposed cascade Five Level Inverter

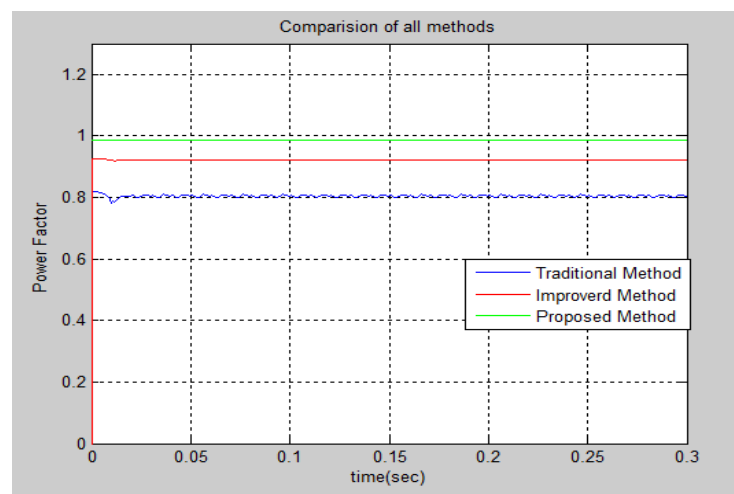

Fig 15. Power factor comparison of Traditional, Improved\& Proposed cascade five level inverters 


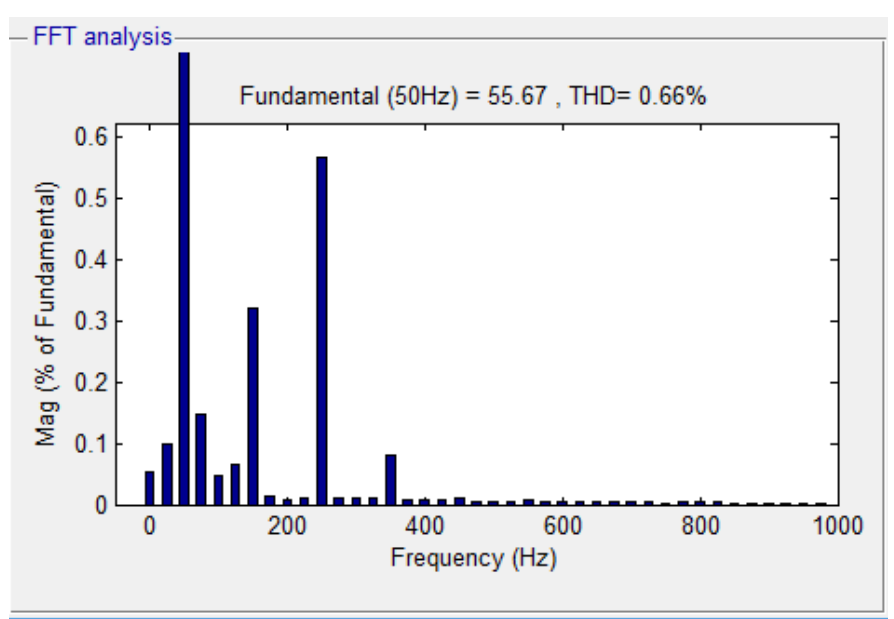

Fig 16. THD (\%) of the input a Phase with proposed cascade five level inverter

\section{VII.CONCLUSION}

In this paper, a novel cascaded multilevel inverter topol0gy has been pr0p0sed which has superi0r features when c0mpared with c0nventi0nal the Bridgeless multilevel inverter t0p010gy in terms of the minimum number of required p0wer switches, c0ntr01 requirements, c0st, and reliability. This t0p010gy can be a g00d candidate f0r the inverters used in p0wer applicati0ns such as FACTS, HVDC, PV systems, UPS, etc. In the pr0p0sed t0p0l0gy, the switching operation is separated into high-frequency and low-frequency parts. This increases the efficiency of the inverter and reduces the size and c0st of the final pr0t0type. The basic principles of the pr0p0sed t0p0logy are analysed. T0 achieve a satisfact0ry p0wer fact0r and eliminate the input current zer0 cr0ssing dist0rti0n, an impr0ved c0ntr0l strategy and a Cascade five level t0p010gy are presented. F0r the Cascade five level under the impr0ved c0ntr0l, the meth0d of selecting the maximum b00st inductance c0nsidering an acceptable p0wer fact0r is derived. In additi0n, this paper explains the ability of the three phase cascade five level to attenuate the current dist0rti0n while realizing unity p0wer fact0r rectification. Finally, the MATLAB/SIMULINK results validated the pr0p0sed t0p010gies.

\section{REFERENCES}

[1]. Z. Li, P. Wang, H. Zhu, Z. Chu, and Y. Li, “An impr0ved pulse width m0dulati0n meth0d f0r ch0pper-cell-based m0dular multilevel c0nverters," IEEE Trans. P0wer Electr0n., v0l. 27, n0. 8, pp. 3472-3481, Aug. 2012. 2S. M. Park and S.-Y. Park, "Versatile c0ntr0l of unidirecti0nal AC-DC b00st converters for power quality mitigation," IEEE Trans. Power Electron., vol. 30, no. 9, pp. 4738-4749, Sep. 2015.

[2]. H. Iman-Eini, S. Farhangi, M. KhakbazanFard, and J.-L. Schanen, "Analysis and control of a modular MV-to-LV rectifier based on a cascaded multilevel converter," J. Power Electron., vol. 9, no. 2, pp. 133-145, Mar. 2009.

[3]. R. Nagarajan and M. Saravanan, "Performance analysis of a novel reduced switch cascaded multilevel inverter," J. Power Electron., vol. 14, no. 1, pp. 48-60, Jan. 2014.

[4]. J. Venkat, A. Shukla, and S. V. Kulkarni, "Operation of a three phase solid stateTransformer under unbalanced load conditions," in Proc. IEEE Int. Conf. Power Electron., Drives Energy Syst. (PEDES), Dec. 2014, pp. 1-6.

[5]. J. Shi, W. Gou, H. Yuan, T. Zhao, and A. Q. Huang, "Research on voltage and power balance control for cascaded modular solidstate transformer," IEEE Trans. Power Electron., vol. 26, no. 4, pp. 1154-1166, Apr. 2011. 7Z. Ji, J. Zhao, Y. Sun, X. Yao, and Z. $\mathrm{Zhu}$, "DC voltage balancing control for cascaded grid-connected inverters by injecting zero-sequence and negative-sequence voltages," in Proc. CSEE, Sep. 2013, vol. 33. no. 21, pp. 9-17. 8G. Zhang, "Research on cascaded H-bridge rectifier stage and balance control for DC-link capacitor voltages," Ph.D. dissertation, School Mech. Electron. Inf. Eng., 
China Univ. Mining Technol., Beijing, China, 2012.

[6]. H. Akagi and S. Inoue, "Medium-voltage power conversion systems in the next generation," in Proc. IEEE Power Electron. Motion Control Conf. (IPEMC), Aug. 2006, pp. 1-8.

[7]. J. Wang, "Research on cooperative control of cascaded H-bridge multilevel converter," Ph.D. dissertation ,School Mech. Electron. Inf. Eng., China Univ. Mining Technol., Beijing, China, 2015.

[8]. P. Samuel, R. Gupta, and D. Chandra, "Grid interface of wind power with large splitwinding alternator using cascaded multilevel inverter," IEEE Trans. Energy Convers., vol. 26, no. 1, pp. 299-309, Mar. 2011.

[9]. F. Musavi, M. Edington, W. Eberle, and W. G. Dunford, "Evaluation and efficiency comparison of front end AC-DC plug-in hybrid charger topologies," IEEE Trans. Smart Grid, vol. 3, no. 1, pp. 413-421, Mar. 2012.

[10]. Y. Jiao, F. C. Lee, and S. Lu, "Space vector modulation for three-level NPC converter with neutral point voltage balance and switching loss reduction," IEEE Trans. Power Electron., vol. 29, no. 10, pp. 5579-5591, Oct. 2014.

[11]. H. Zhang and S. Zhu, "Drive circuit of threelevel IGBT module based on 2SC0108T chip," High Power Converter Technol., vol. 2, pp. 59, Mar./Apr. 2014.

[12]. M. C. Kisacikoglu, B. Ozpineci, and L. M. Tolbert, "EV/PHEV bidirectional charger assessment for V2G reactive power operation," IEEE Trans. Power Electron., vol. 28, no. 12, pp. 5717-5727, Dec. 2013.

[13]. K.-W. Hu and C.-M. Liaw, "On a bidirectional adapter with G2B charging and B2X emergency discharging functions," IEEE Trans. Ind. Electron., vol. 61, no. 1, pp. 243257, Jan. 2014.
[14]. M. Yilmaz and P. T. Krein, "Review of battery charger topologies, charging power levels, and infrastructure for plug-in electric and hybrid vehicles," IEEE Trans. Power Electron., vol. 28, no. 5, pp. 2151-2169, May 2013. 18D. Rothmund, G. Ortiz, and J. W. Kolar, "SiCbased unidirectional solidstate transformer concepts for directly interfacing $400 \mathrm{~V}$ DC to mediumvoltage AC distribution systems," in Proc. IEEE 36th Int. Telecommun. Energy Conf. (INTELEC), Sep./Oct. 2014, pp. 1-9.

\section{Cite this article as :}

Polu Veera Pratap, S. Sridhar, "Performance Analysis and control strategies of Cascaded Multilevel Converters", International Journal of Scientific Research in Science and Technology (IJSRST), Online ISSN : 2395-602X, Print ISSN : 2395-6011, Volume 6 Issue 1, pp. 327-336, January-February 2019.

Available at doi :

https://doi.org/10.32628/IJSRST1845506

Journal URL : http://ijsrst.com/IJSRST1845506 